Progress Report 10/28/97

David E Salt

EMSP grant \#DE-FG07-96ER20251

\title{
MOLECULAR DISSECTION OF THE CELLULAR MECHANISMS INVOLVED IN NICKEL HYPERACCUMULATION
}

\section{PROJECT SUMMARY}

Phytorcmediation, the use of plants for environmental cleanup of pollutants, including toxic metals, holds the potential to allow the economic restoration of heavy metal and radionuclide contaminated sites. A number of terrestrial plants are known to naturally accumulate high levels of metals in their shoots (1-2\% dry weight), and these plants have been termed metal-hyperaccumulators. Clearly, the genetic traits that determine metal-hyperaccumulation offers the potential for the development of practical phytoremediation processes. Our long-term objective is to rationally design and generate plants ideally suited for phytoremediation using this unique genetic material.

Initially, our strategy will focus on isolating and characterizing the key genetic information needed for expression of the metal-hyperaccumulation phenotype. Recently, histidine has been shown to play a major role in Ni hyperaccumulation. Based on this information we propose to investigate. at the molecular level, the role of histidine biosynthesis in Ni hyperaccumulation in Thlaspi goesingense, a $\mathrm{Ni}$ hyperaccumulator species.

- We will clone key genes involved in histidine biosynthesis

- We will characterize their transcriptional and post transcriptional regulation by histidine, Ni and other heavy metals, and

- We will determine if any of these genes are essential and sufficient for $\mathrm{Ni}$ hyperaccumulation by their expression in the non-hyperaccumulator Arabidopsis thaliana.

\section{PROGRESS IN CLONING OF KEY GENES INVOLVED IN HISTIDINE BIOSYNTHESIS}

In order to clone genes from the Ni hyperaccumulator $T$. goesingense a cDNA library (2TriplEx) has been constructed. This library has successfully been used to clone, by functional complementation in $\boldsymbol{E}$. coli mutants, genes coding for phoshoribosyl ATP transferase (ATP PRT), imidazoleglycerol phosphate dehydratase (IGPD), and histidinol dehydrogenase (HD), three key genes involved in histidine biosynthesis in plants (Fig 1). Using Southern analysis we have confirmed the presence of these three genes in the $T$. goesingense genome. All three cDNA sequences have been submitted to GenBank. To our knowledge these represent the first genes to be cloned from a metal hyperaccumulating plant.

All three T. goesingense genes (THGl, THBl, THDl) have been engineered into an $\boldsymbol{E}$. coli expression vector for expression and purification of the recombinant proteins. Once purified, these proteins will be used to study the allosteric regulation of the enzyme activities by nickel. Purified proteins will also be used to raise antibodies for quantification 
of the proteins in plant tissues after nickel exposure . Using THGI, THB1 and THDl as probes we are now in the process of studying the transcriptional regulation of these genes by nickel, using Northern analysis, and these studies should be complete in the next $3-6$ months.

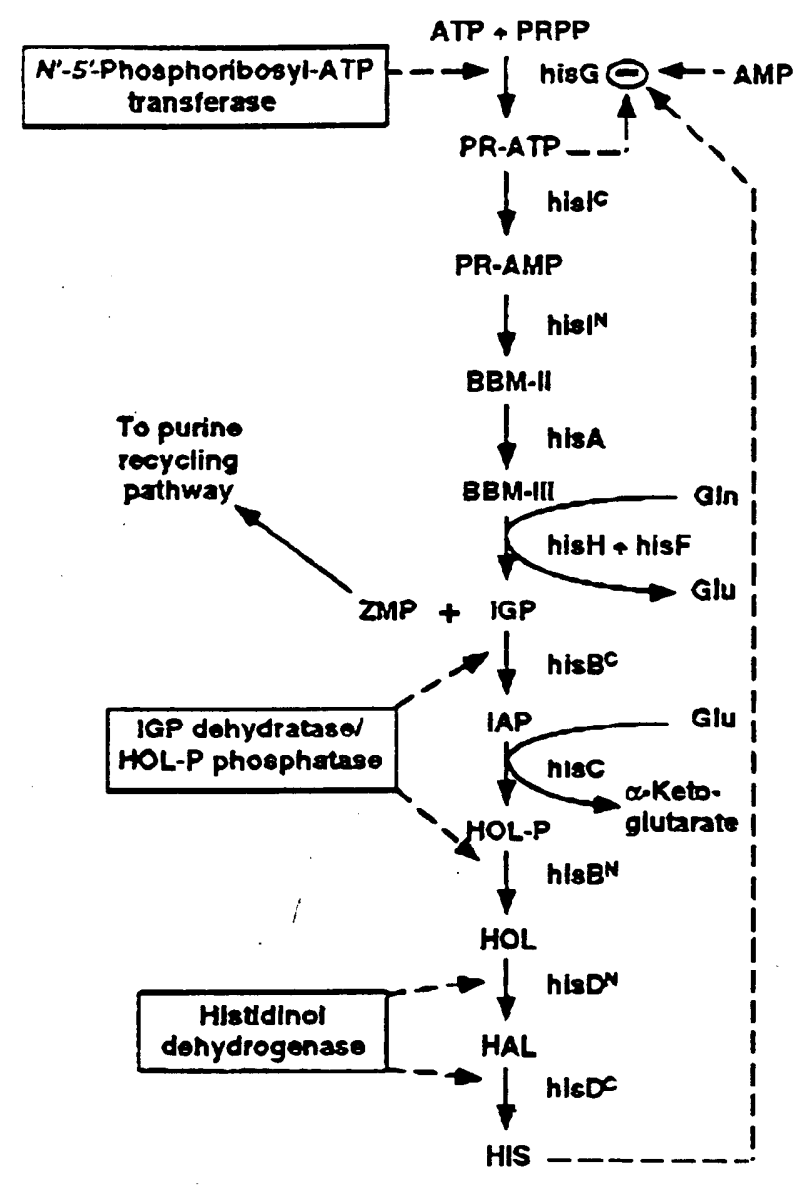

Figure 1. Histidine biosynthetic pathway

\section{Details of Histidine Biosynthetic} Genes Cloned from $T$. goesingense

Phoshoribosyl ATP transferase: This is the first example of a plant ATP-PRT gene and we have termed it THGI (Thlaspi HisG 1). A comparison of the sequence of THGl with those available in GenBank showed that the top 17 sequences having significant homology (approximately 30\% identity at the amino acid level) were all genes encoding ATP-PRT enzymes from various microorganisms (Fig 2A). Analysis of the THGl sequence uncovered the existence of an N-terminal chloroplast targeting sequence approximately 30 amino acids long. This is consistent with biochemical evidence that suggests histidine biosynthesis occurs in the chloroplast.

\section{Imidazoleglycerolphosphate} dehydratase: Several genes encoding for IGPD's have been cloned from plants including wheat, pea-and Arabidopsis The amino acid sequence of the gene we cloned from $T$. goesingense (THB1)

shows 85 - 90\% sequence identity to the other known plant genes (Fig 2B). Sequence analysis of THB1 also identified a chloroplast targeting sequence of approximately 50 amino acids. This is consistent with that found for THG1, and localization of histidine biosynthesis in the chloroplast The putative chloroplast targeting sequence in THB1 is less conserved than the main coding region, as would be expected for a targeting sequence (Fig 2 B).

Histidinol dehydrogenase: The amino acid sequence of the $T$. goesingense gene (THDI) encoding HD, cloned by us, shows $89 \%$ identify to a HD gene previously cloned from cabbage (Brassica oleracea) (Fig 2C). Analysis of the amino acid sequence of THDl identified an $\mathrm{N}$-terminal targeting sequence which may be involved in targeting to the chloroplast. Chloroplast targeting of THDl is consistent with that found for both THG1 
Progress Report 10/28/97

David E Salt

EMSP grant \#DE-FG07-96ER20251

and THB1. The putative chloroplast targeting sequence of THD1 is less conserved than the main coding region, as observed in THB1, and as would be expected for a targeting sequence (Fig $2 \mathrm{C}$ ).

SIMILARTTY SCALE (\%)

6.

\begin{tabular}{llllllllll}
\hline Nothing detected & 20 & 30 & 40 & 50 & 60 & 70 & 80 & 90 & 100
\end{tabular}

A.

T. goesingense

ATP-phosphoribosyl transferase (ATP-PRT)

0

A

S. pombe

3.3.

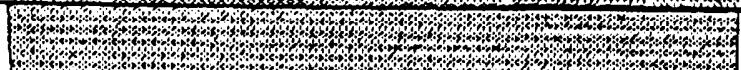

3. $10 \%$

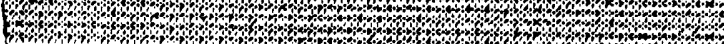

0

300

B.

T. goesingense r

Imidazoleglycerol phosphate dehydratase (IGPO)

250

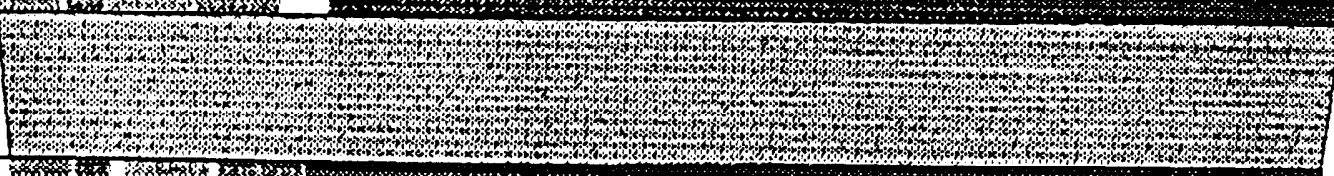

A. thaliana

0

c.

T. goesingense

Histidinol dehydrogenase (HD)

450

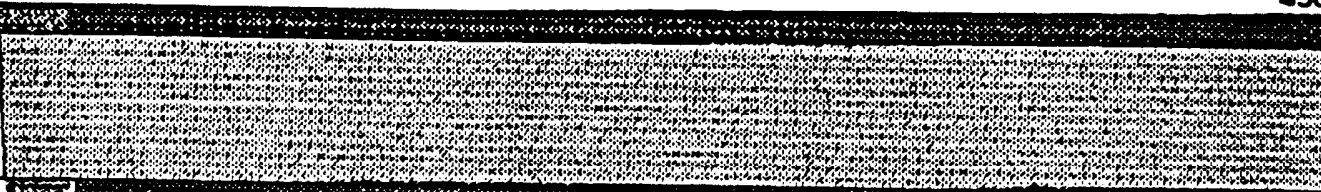

8. oleracea

0

Figure 2. Amino acid aliments of histidine biosynthetic genes cloned from the Ni hyperaccumulator $T$. goesingense with the same genes from other species. 